\title{
A Student Based Biometric Application
}

\author{
Renshan Jia1)
}

\begin{abstract}
The latest developments in technology especially in the field of computer science provided a new scope for IoT (internet of things). IoT makes use of sensors and the internet it is a combination of hardware and software functioning together. The identification of a person is becoming important day today due to various reasons. Some reasons may be security reasons while some others reason may be to identify the persons easily rather than asking them individually which is a time taking process. Hence, more importance was given to making the identification of a person more automatic and the process can be made easier. The important factors to be considered for identifying a person are the facial expressions or structures, biometric and the iris of a person. Hence, in the current article, an attempt has been made to automate the attendance system automatically by collecting the fingerprints of the students and the attendance of students is made automatically. This application was being developed with the IoT model and the results are more encouraging.
\end{abstract}

Keywords : Students, Attendance, Arduino, IoT, Circuit, Android, Biometric

\section{Introduction}

The identification of a human in the current days is gaining importance and it is the currently developed technology or the applications used worldwide such that for various reasons. Some of those reasons are security reasons. The identification of a person at airports, identifying the individuals for purchasing any item or in some cases identifying the person for providing or issuing the bank loans, etc. Some other cases like the identification of a person for providing the secured bank lockers and also for providing security to office entering and other private restricted areas[1-4]. All these are some of the applications where the identification of the person is required, but also some other areas also there where the requirement for identifying a person is more vital and more important. Several methods have been used so far to identify a person based on the appearance of a person. It is very important to identify the person who is entering an airport. If the authorities will check person by person, it will more time and the queue lines with the passengers will increase or the other problem can be resolved is to

Received(August 07, 2019), Review Result(1st: September 03, 2019, 2nd: October 24, 2019), Accepted(November 15, 2019)

1) (Professor) Heilongiiang Collage of Business and Technology, China

email: sakura2370@163.com 
increase the number of people at checking places to make it faster. Hence, there is a need for a system such that to identify the persons who are entering and who are leaving the airports with automatic face recognition systems. Nowdays, all the airports are going towards this sort of application. Which reduces the cost of operations and will make the system more transparent.

The other applications that the identification system requires are land registrations. The same land can be occupied by different people and can claim that to particular will belongs to them. To overcome such cases, the utilization of the individual persons who are actual owners will have to produce their iris, thumb and also facial recognition such that the unauthorized person's occupation of some lands can be reduced. It is also important to reduce or identify the persons who are entering the restricted areas. Hence, for anyone using this sort of application, the entering of unauthorized persons into the restricted areas can be restricted. For implementing these sorts of applications, biometric applications are one of the most famous and important solutions current day. The utilization of IoT technology for these applications will increase the results more and more and also can increase the security of current-day applications[5].

For any biometric system, several points are factors to be considered for their smooth functioning. They are retina, iris, signature, voice, face or facial, geometry, etc. In most cases, all these factors for an individual person will have unique features and if we collect these factors of all the people, then the implementation and the identification of the identification systems will have more accurate functioning. All these factors are unique for a person or individual throughout their life and cannot be changed. This may be taken as the good and most important point to be considered. Fingerprints can be considered unique because they may not change from time to time for any person and moreover, no two persons may have the same patterns in their palms. If we consider the palm of a person, the gaps between the ridges and the differences cannot be calculated or made artificially by any person, hence considering these factors may give us very good results and be used these systems more accurately and more in number.

The biometric attendance system is designed to provide ease of access to the daily attendance and replace the manual attendance with server automated attendance. It uses a fingerprint scanner to detect the student's fingerprint and update the attendance database after validating the fingerprint of the student[6][7]. This will switch the whole attendance system in colleges and organizations. In the earlier days, the RFID tags were being used and the students can make the system cheat by simply carting the tags of other students and the attendance for 
those absent students also the attendance will be processed. To overcome these issues, this current model of the system was developed. Attendance of a student is most important for any institute. The performance of the student lies in the number of days that a student is attending the classes and how he is performing. Hence, in order to make it more genuine and transparent an attempt has been made to make an attendance system that too an automated system with the help of their biometrics. Once the student enters into the institute and gives their biometric, automatically the attendance of the student will be updated. By using the IoT technology and other sensor networks and other sensors, an attempt has been made to develop and work on an attendance system. The proposed model is the fingerprint-based model and the hardware used here is the Arduino UNO and other devices for collecting the fingerprints and processing them.

In previous days people use to register their attendance in a sheet or a book and later they will calculate the attendance percentage. In this process, there is less accuracy in attendance. At present days people use the biometric system[8]. The biometric is refer to the identification and verification of a person based on physical or behavior through particular techniques. Biometric technologies can define as an automated system of recognizing a person based on the person's physical or behavioral characteristics. Nowadays, advanced biometric technology may be an option for identification applications rather than using the physical card, documents, passwords, or ID numbers. The basic processes inside the biometric technologies are to capture and store the information at the enrolment stage and then compare it at a later verification stage[9][10].

\section{Existing System}

There are existing systems for the product we have developed but most of them are using embedded systems which are costly to build, we are using Arduino which is very cost-efficient and easy for further updates.

\section{Disadvantages of the Existing Model}

The disadvantage of using this product is we cannot provide more storage for the fingerprints using EP-ROM.

\section{Proposed System}

Now a day in the education system or in educational institutions, attendance monitoring and 
providing attendance to the students had become more important. It is gaining more importance day today. As cheating in society is increasing from day to day, educational institutions cannot depend on human and also on RFID tags. Nowadays, most of the students in the colleges had given the tags. However, the students who are not attending the colleges regularly. Not only for the colleges, but it is also important for the local governments to provide financial assistance to the students who are poor and the governments are providing such assistance based on the attendance of the students. Earlier RFID tags were being used for students. Nowadays the cheating was increased with these tags, hence an attempt or idea was tried to implement the attendance based on the fingerprint selection of the students. The features of the current developed system were as follows,

It overcomes all the problems in the existing system and provides accuracy and efficiency. This is a perfect/optimal solution for the accuracy of attendance. The features of our product are briefly described below:

A Completely automated system helps the customer to use the product with ease.

A student cannot give the attendance of another person.

The student need not carry any RFID cards also and they can record their attendance by their fingerprint, which is an efficient and secured method.

In order to implement the current model, first, the authors need to register themselves in the current application. Every student and other staff in the institute would register themselves by providing their particular details in the system. Once, the registration process is completed, the details of all the users are entered and stored in the database. The next process of the model is to give the input to the system by giving the fingerprints of the users. Based on the given input data, the machine will work on the given input and it will register for the user.

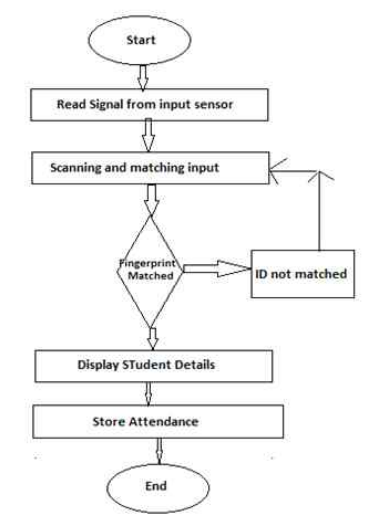

[Fig. 1] Registration Process Model 
The architecture model of the proposed model can be observed as,

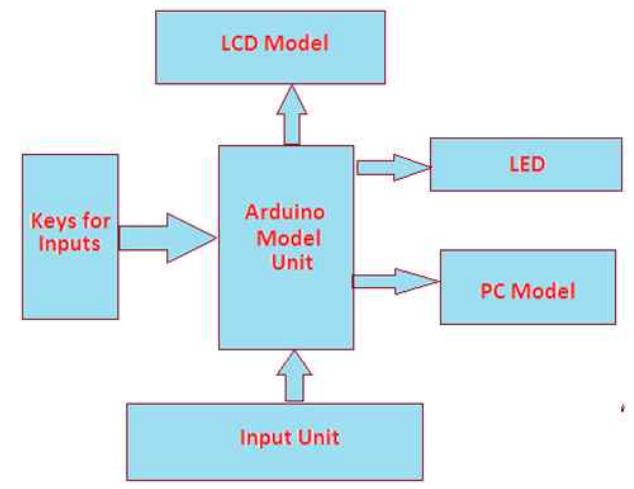

[Fig. 2] Architecture of the Current Model

In the current model of the attendance system, a sensor module was used which will work on the fingerprints of a particular user. Based on the fingerprints of the user, the machine or the model will identify and update the database. It is also used to identify whether the biometric was given by the actual person or the fake person. Based on the input data, the machine will provide the decision of whether the permission for access or to deny will be decided. Several options were given in the working of the current unit. The moving of the cursors to left, right, and top/down also given in the current model. The enrolment to the current system at any time was also given with proper admin permission and with proper utilization of the conditions for the registration process. In order to view the tasks or the operations going on in the unit, a display unit also fixed in the module such that to observe the operations that were being performed on this module. A timer was also given such that to record the events of things that were taking place in the unit will also be recorded. The number of people or the users can be increased at any point in time and also can be deleted at any point in time.

Working on this biometric attendance system project is fairly simple. First of all, the user needs to enroll fingerprints of the user with the help of pushbuttons. To do this, the user needs to press ENROLL key and then LCD asks for entering the ID for the fingerprint to save it in memory by ID name. So now the user needs to enter ID by using UP/DOWN keys. After selecting ID, the user needs to press an OK key (DEL key). Now LCD will ask to place a finger over the fingerprint module. Now user needs to place his finger over the fingerprint module and then the module takes a finger image. Now the LCD will say to remove finger from fingerprint module, and again ask to place finger again. Now user needs to put his 
finger again and the module takes an image and converts it into templates and stores it by selected ID into the fingerprint module's memory. Now the user will be registered and he/she can feed attendance by putting their finger over the fingerprint module. By the same method, all the users will be registered into the system.

\section{The Major Advantages of the Current Module are as follows,}

1. Security: Security is of main concern in any locking system. When the lock and Key system is concerned, security is very poor compared to other improved security systems as any key can be duplicated at any time. A password authentication system provides pretty good security compared to a lock and key system but there are chances for intruders to hack the database of passwords and break the system. FID card provides good authentication compared to the above system. This card can be misused when caught into the hands of intruders. On the other hand, an intelligent door locking system provides complete security as a fingerprint is a unique identifier for each user. It is impossible for fingerprint duplication. Hence this system provides optimal security compared to other systems.

2. Cost: When a new system is launched in the market, it is considered only when it cost-effective than other existing systems. The lock and key system is of less cost than all other systems and is available to all users. Both password and RFID authentication is more costly and also provide considerable security than a lock and key system. On the other hand, our system offers extreme security for optimum cost.

3. Usability: Success in the system lies in the comfort of using it. Users are mode adapted to the usage of the lock and key system. On the other hand, a password authentication system requires the user to remember the complex passwords created by the user. FID system requires the user to maintain the RFID card safely without any damage. On the other hand, an intelligent door locking system is comfortable to use as it uses a fingerprint for authenticating the user. Hence this system is user-friendly compared to all other systems.

\section{Implementation and Results}

The actual implementation was done for the current model by the kit that was developed with the help of several hardware units. Several units are collected for inputs as the fingerprint scanner is collected for collecting the inputs from the users. The other units like the Arduino unit was selected for processing of the entire internal process were selected and the data was given and stored and processed and the results were displayed. For displaying the output 
results, an LCD screen and LED unit were also selected such that to provide display and also for operating the internal tasks.

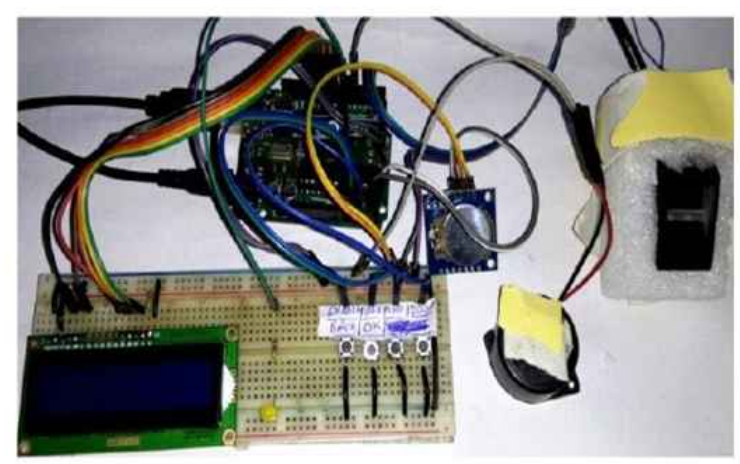

[Fig. 3] The Hardware Kit Module Model

The above screenshot Fig 4.1 represents the prototype of the biometric attendance system. The components mentioned in the screenshot are a 2-pin switch, Arduino microcontroller, LCD screen, fingerprint sensor, resistors, buzzer, breadboard, and RTC module.

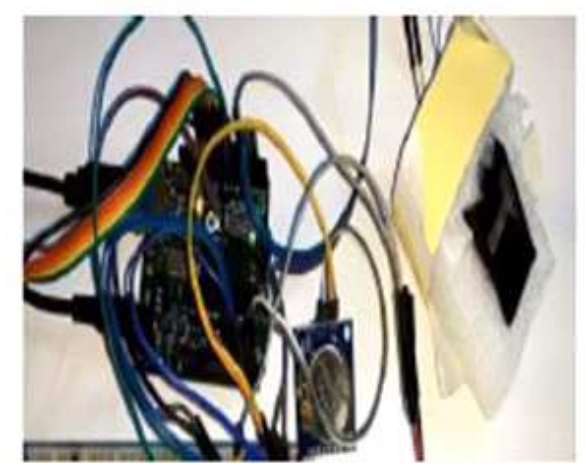

[Fig. 4] Arduino Model

The [Fig. 4] shows the Arduino connections to fingerprint. Every component in this process will be connected to the Arduino directly or indirectly to perform the operations. 


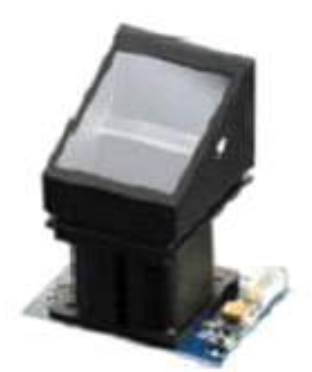

[Fig. 5] The Input Scanner Model

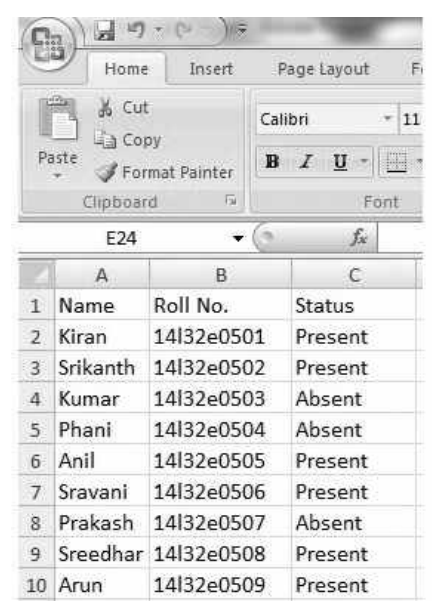

[Fig. 6] Student Details Output Sample

The [Fig. 5] represents the fingerprint sensor R305, this module can store the fingerprints up to 1000 fingerprints. The functionality of this optical fingerprint sensor is, that scanners use an LED light to illuminate the finger. The sensor detects and creates the fingerprint image by determining the light and dark areas created by the fingerprint ridges. The scanning process starts when the individual places his finger on the glass plate which is known as a touch surface. This captured image is then processed and different features like patterns and characteristics of the fingerprints are identified and they are converted into a digital template which is stored in the fingerprint database. An example of student attendance was taken for a sample of ten students and the result was given in [Fig. 6].

\section{Conclusions}

In the current model, the authentication system can be considered one of the best of the 
existing models. The biometric cannot be made duplicate and the duplication of things is very few. More chances are there to get the model to be worked accurately in most of the cases or the scenarios. By using the current model, the attendance can be taken correctly in most of the cases and the output of the model will give us detailed data about the number of students who got absent and got present for that particular day. Also, it provides the data about the full details of the students who got absent like the name of the student with roll number. As a result of this, the users can find the details very easily, and also the operation of the unit is also very easy. The results obtained from the current model are satisfying and can be used for furthermore applications.

\section{References}

[1] Jae-Woo Kim, Jeong-Hyo Park, Moon-Seog Jun, A Design of Smart Banking System using Digital Signature based on Biometric Authentication, Journal of Korea Academia-Industrial cooperation Society, (2015), Vol.16, No.9 pp.6282-6289, DOI: 10.5762/KAIS.2015.16.9.6282

[2] Hyung-Woo Lee and Yeong-Joon Park, A Design and Implementation of User Authentication System using Biometric Information, Journal of Korea Academia-Industrial cooperation Society, (2010), Vol.11, No.9, pp.3548-3557, UCI: G704-001653.2010.11.9.024

[3] SungHyun Yun, The Biometric based Convertible Undeniable Multi-Signature Scheme, Journal of Korea Academia-Industrial Cooperation Society, (2010), Vol.11, No.5, pp.1670-1676, UCI: G704-001653.2010.11.5.038

[4] SujayPatole, YatinVispute, Automatic Attendance System Based On Face Recognition, International Journal of Innovative Research in Science, Engineering and Technology, (2017), Vol.6, No.8, pp.16009-16016, DOI: 10.15680/IJIRSET.2016.0608042

[5] Syed Ibrahim, Automatic Attendance System Using Facial Detection and Recognition Technique, International Journal of Engineering and Computer Science, (2018), Vol.7, No.10, pp.24372-24376.

[6] Mohit Bais, Deeksha Rawat, Gunjeet Kaur, Biometric Attendance System Circuit, International Journal of Engineering Applied Sciences and Technology, (2016), Vol.1, No.6, pp.195-199.

[7] Priyanka Sahare, Pranali Gaikwad, Snehal Narule, Nutan Thakre, Puja Chandekar, RFID Technology Based Attendance Management System, International Journal of Engineering and Computer Science, (2017), Vol.6, No.3, pp.20458-20463.

[8] Joseph Elijah, Amit Mishra, M. Usman Gana, Engr. Mathew Chukwu Udo, Abiodun Musa Aibinu, Staff Monitoring System Using Biometric, International Journal of Engineering and Computer Science, (2017), Vol.6, No.5, pp.21448-21458, DOI: $10.18535 /$ ijecs/v6i5.43

[9] Purnima Singh, Archana Gautam, Km. Jyotsana, Diksha Srivastav, Aditya Kumar Singh, Students Record System using Radio Frequency Technique, International Journal of Engineering and Computer Science, 
A Student Based Biometric Application

(2016), Vol.5, No.5, pp.16447-16452.

[10] Aditya Desai, Tejashri Desai, Hemangi Salunkhe, Sumitra Teli, Praveen Patil, Effective Student Attendance System, International Research Journal of Engineering and Technology, (2018), Vol.5, No.10, pp.1205-1206. 